\title{
Comparisons between Standard and Extended Pelvic Lymph Node Dissections During Radical Cystectomy in Patients with Bladder Cancer: Emphasis on Staging Ability and Perioperative Complications
}

\author{
Sang Eun Lee, Hakmin Lee, Hyun Hwan Sung, Seong II Seo, Seong Soo Jeon, \\ Hyun Moo Lee, Han-Yong Choi, Byong Chang Jeong \\ Department of Urology, Samsung Medical Center, Sungkyunkwan University School of Medicine, Seoul, Korea
}

\begin{abstract}
Purpose: We compared the staging ability and early complications of standard and extended pelvic lymph node dissection (sPLND and ePLND, respectively) in patients with localized bladder cancer during radical cystectomy.

Materials and Methods: We prospectively collected and analyzed the data of 261 patients who underwent radical cystectomy for localized bladder cancer. The resected lymph nodes were categorized according to anatomic locations and were carefully inspected by an experienced pathologist. The perioperative complications were classified using the Clavien-Dindo classification system.

Results: The 2 groups showed no significant differences in preoperative characteristics except for preoperative clinical stage $(p=0.015)$. There were no significant differences in pathologic outcomes including pathologic stage, positive surgical margin, and lymphovascular invasion (all $p>0.05$ ), but the sPLND group showed a significantly higher cellular grade $(p<0.001)$. The ePLND group showed a higher number of removed lymph nodes than the sPLND group $(p=0.015)$ and a higher rate of positive lymph node invasion $(35.8 \%$ vs. $28.9 \%)$. There were no significant differences in complication rates according to the extent of lymph dissection and urinary diversion type, respectively ( $p=0.063$ and $p=0.486$ ).

Conclusions: The ePLND showed more accurate nodal staging ability with comparable complication rates when compared to SPLND in patients who underwent radical cystectomy for localized bladder cancer. A further, larger prospective study is needed to confirm the result of the present study. (Korean J Urol Oncol 2016;14:124-129)
\end{abstract}

Key Words: Urinary bladder neoplasms $\cdot$ Radical cystectomy $\cdot$ Lymph node excision

Received June 20, 2016, Revised August 15, 2016,

Accepted August 18, 2016

Corresponding Author: Byong Chang Jeong

Department of Urology, Samsung Medical Center, Sungkyunkwan University School of Medicine, 81 Irwon-ro, Gangnam-gu, Seoul 06351, Korea

E-mail: bc2.jung@samsung.com

Tel: +82-2-3410-3557, Fax: +82-2-3410-6992

\section{INTRODUCTION}

More than 70,000 patients are newly diagnosed with urothelial cancer of the bladder and more than 14,000 patients die of the condition per year in the United States. ${ }^{1}$ About $40 \%$ of the patients were found to have muscle invasive bladder cancer (MIBC), with a $25 \%$, lymph node metastasis rate. ${ }^{2}$ To date, radical cystectomy with pelvic lymph node dissection (PLND)

(i) (1) This is an Open Access article distributed under the terms of the Creative Commons Attribution Non-Commercial License (http://creativecommons.org/licenses/by-nc/4.0/) which permits unrestricted non-commercial use, distribution, and reproduction in any medium, provided the original work is properly cited. 2016 (C) Copyright The Korean Urological Oncology Society and The Korean Prostate Society. All Rights Reserved. 
has been the standard treatment in patients with MIBC if there is no evidence of distant metastasis. ${ }^{3}$ Lymph node dissections during radical cystectomy have been regarded as important for both disease staging and therapeutic benefits.

The main discussion regarding PLND in the context of radical cystectomy is the optimal extent of PLND. ${ }^{4}$ Evidence shows that higher number of collected lymph nodes is associated with superior survival outcomes. ${ }^{5,6}$ However, there is no consensus regarding the optimal extent of PLND in patients with clinically organ-confined MIBC. ${ }^{7}$ Several previous studies had evaluated this conundrum, but they were mostly retrospectively designed, with only a small number of patients, and were mostly evidence level 3 or 4 studies. $^{8-11}$ Neither the European Association of Urology nor the American Urological Association provides clear guidelines regarding the optimal anatomic extent of PLND in dealing with these localized MIBC patients. ${ }^{12}$ More importantly, the postoperative complications after standard and extended PLND (sPLND and ePLND, respectively) were poorly investigated to date.

We compared the staging accuracy between ePLND and SPLND in patients with localized MIBC treated during radical cystectomy with PLND in our institution. We also evaluated early ( $\leq 30$ days) postoperative complications according to PLND extent after surgery.

\section{MATERIALS AND METHODS}

After the approval of Samsung Medical Center Institutional Review Board (approval number: 2016-002), we prospectively collected information on 293 patients who underwent PLND for nonmetastatic bladder cancer from January 1991 to December 2014 in single tertiary center located in South Korea. After further exclusion of 27 patients (other malignancy, $n=12$; concurrent nephron-ureterectomy, $\mathrm{n}=6$; previous pelvic radiation therapy, $n=11$; incomplete information, $n=3$ ), we finally analyzed 261 subjects. Clinical and pathologic information were acquired from the review of medical records. The extent of PLND was decided by each corresponding surgeon according to preoperative clinical and pathologic information. Pathologic staging was based on the American Joint Committee of Cancer TNM classification, and histological grade was assessed according to the World Health Organization International Society of Urological Pathology classification. ${ }^{13,14}$ Lymphovascular invasion or surgical margin status were also evaluated by patho- logic assessment.

The specimens from PLND were categorized into 5 groups according to the resected anatomic region: (1) external iliac, (2) obturator (including the internal iliac region), (3) common iliac, (4) presacral, and (5) para-aortic. The sPLND included the external iliac and obturator regions. ePLND was defined as sPLND plus the following extra regions: common iliac, presacral, and para-aortic areas. Each specimen was analyzed separately according to the anatomic origin of the resection. Information on early postoperative complications ( $\leq 30$ days) was also acquired from the review of medical records and categorized according to the Clavien-Dindo classification. ${ }^{15}$ The patients' clinical and pathologic characteristics were compared using independent t-test and chi-square test between PLND groups. Statistical analysis was performed using the IBM SPSS Statistics ver. 19.0 (IBM Co., Armonk, NY, USA). A p-value $<0.05$ was regarded as statistically significant, and all p-values were two-sided.

\section{RESULTS}

The preoperative clinical and pathologic characteristics of all patients are described in Table 1 . When we compared the perioperative characteristics according to the PLND method, there were no significant differences between the groups, but the patients who underwent SPLND showed significantly worse clinical stage before surgery $(p=0.015)$. The pathologic outcomes including pathologic stages, lymphovascular invasion, surgical margin status and operation time were not significantly different between the 2 groups, but the sPLND group showed a significantly higher grade than the ePLND group $(\mathrm{p}=0.001)$. The number of collected lymph nodes were significantly higher in the ePLND group than in the sPLND group ( $\mathrm{p}=0.015)$.

Comparison of detection rates of positive lymph nodes according to the PLND method showed that the ePLND group had a higher percentage of patients with lymph node invasion than the sPLND group ( $35.8 \%$ vs. $28.9 \%$, respectively) (Table 2). However, the percentages of positive lymph nodes per total number of removed lymph nodes were the same between the 2 groups (both $8.2 \%$ ). Subsequently, we evaluated the rate of lymph node invasion according to the region of the lymph nodes in patients who underwent ePLND (Fig. 1). A low but nonnegligible percentage of patients had lymph node invasions in the ePLND regions (common iliac, presacral, and para-aortic) 
Table 1. Perioperative characteristics of all patients and subgroups according to extent of lymph node dissection

\begin{tabular}{|c|c|c|c|c|}
\hline Characteristic & Entire patients $(n=261)$ & SPLND $(n=166)$ & EPLND $(n=95)$ & p-value \\
\hline Age (y) & $66(59-72)$ & $66(59-73)$ & $64\left(58^{-71)}\right.$ & 0.180 \\
\hline Male sex, yes & $230(88.1)$ & $142(85.5)$ & $88(92.6)$ & 0.089 \\
\hline Body mass index $\left(\mathrm{kg} / \mathrm{m}^{2}\right)$ & $23.0(20.6-25.1)$ & $23.4(21.0-25.3)$ & $22.4(20.3-24.3)$ & 0.980 \\
\hline Diabetes mellitus & $50(19.2)$ & $31(18.7)$ & $19(20.0)$ & 0.793 \\
\hline Hypertension & $104(39.8)$ & $70(42.2)$ & $34(35.8)$ & 0.311 \\
\hline ASA physical status & & & & 0.518 \\
\hline I & $56(21.5)$ & $32(19.3)$ & $24(25.3)$ & \\
\hline II & $183(70.1)$ & $120(72.3)$ & $63(66.3)$ & \\
\hline$\geq$ III & $22(8.4)$ & $14(8.4)$ & $8(8.4)$ & \\
\hline History of previous pelvic surgery & $22(8.4)$ & $13(7.8)$ & $9(9.5)$ & 0.900 \\
\hline History of intravesical therapy & $36(13.8)$ & $22(13.3)$ & $14(14.7)$ & 0.738 \\
\hline Clinical stage & & & & 0.015 \\
\hline$\leq \mathrm{T} 1$ & $33(12.6)$ & $21(12.7)$ & $12(12.6)$ & \\
\hline $\mathrm{T} 2$ & $94(36.0)$ & $50(30.1)$ & $44(46.3)$ & \\
\hline $\mathrm{T} 3$ & $112(42.9)$ & $83(50.0)$ & $29(30.5)$ & \\
\hline $\mathrm{T} 4$ & $22(8.4)$ & $12(7.2)$ & $10(10.5)$ & \\
\hline Pathologic stage & & & & 0.074 \\
\hline No residual tumor & $10(3.8)$ & $3(1.8)$ & $7(7.4)$ & \\
\hline pT1 & $48(18.4)$ & $26(15.7)$ & $22(23.2)$ & \\
\hline pT2 & $74(28.4)$ & $49(29.5)$ & $25(26.3)$ & \\
\hline pT3 & $96(36.8)$ & $64(38.6)$ & $32(33.7)$ & \\
\hline pT4 & $33(12.6)$ & $24(14.5)$ & $9(9.5)$ & \\
\hline Pathologic grade & & & & 0.001 \\
\hline No residual tumor & $10(3.8)$ & $3(1.8)$ & $7(7.4)$ & \\
\hline Grade 1 & $0(0)$ & $0(0)$ & $0(0)$ & \\
\hline Grade 2 & $35(13.4)$ & $21(12.7)$ & $14(14.7)$ & \\
\hline Grade 3 or CIS & $216(82.8)$ & $142(85.5)$ & 74 (77.9) & \\
\hline Lymphovascular invasion & $99(37.9)$ & $66(39.8)$ & $33(34.7)$ & 0.421 \\
\hline Positive surgical margin & $10(3.8)$ & $4(2.4)$ & $6(6.3)$ & 0.114 \\
\hline No. of resected LN & $17(12.0-23.0)$ & $15.0(10.0-20.3)$ & $22.0(17.0-28.0)$ & 0.015 \\
\hline Operation time (min) & $465.0(397.0-544.5)$ & $453.5(394.8-28.3)$ & $488.0(398.0-65.0)$ & 0.743 \\
\hline
\end{tabular}

Values are presented as number (\%) or median (interquartile range).

SPLND: standard pelvic lymph node dissection, EPLND: extended pelvic lymph node dissection, ASA: American Society of Anesthesiologists, CIS: carcinoma in situ, LN: lymph node.

Table 2. Differences in detection rate of positive lymph node according to extent of lymph node dissection

\begin{tabular}{ccc}
\hline Lymph node dissection & $\begin{array}{c}\text { Patients with positive lymph nodes } \\
\text { (per total number of patients in each subgroup) }\end{array}$ & $\begin{array}{c}\text { Positive lymph nodes } \\
\text { (per total number of removed lymph nodes) }\end{array}$ \\
\hline SPLND & $48(28.9)$ & $207(8.2)$ \\
EPLND & $34(35.8)$ & $181(8.2)$ \\
\hline
\end{tabular}

Values are presented as number (\%).

SPLND: standard pelvic lymph node dissection, EPLND: extended pelvic lymph node dissection.

(mean, 15.8\%). The rates of patients with positive lymph nodes were higher in the SPLND regions (obturator and external iliac) than the ePLND regions (15.8\% vs. $4.0 \%)$. Those trends were also persistent when we calculated the rate of number of positive lymph nodes per total number of resected lymph nodes
(7.8\% vs. $5.9 \%)$.

There were no significant differences in early complication rates between the 2 groups ( $\mathrm{p}=0.063$ ) (Table 3 ) when we categorized the complication according to the Clavien-Dindo classification. The number of high-grade complications 
A

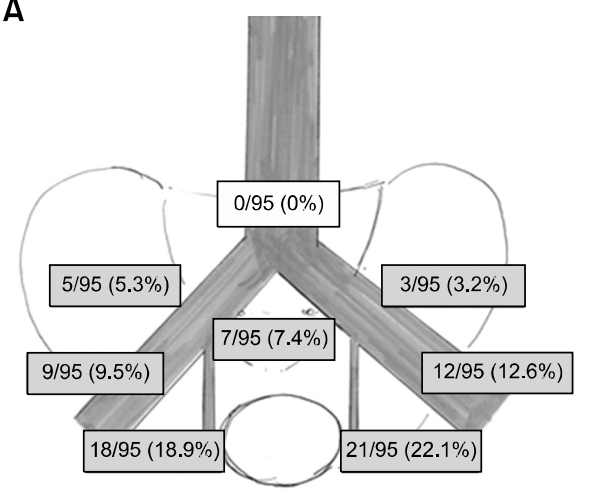

B

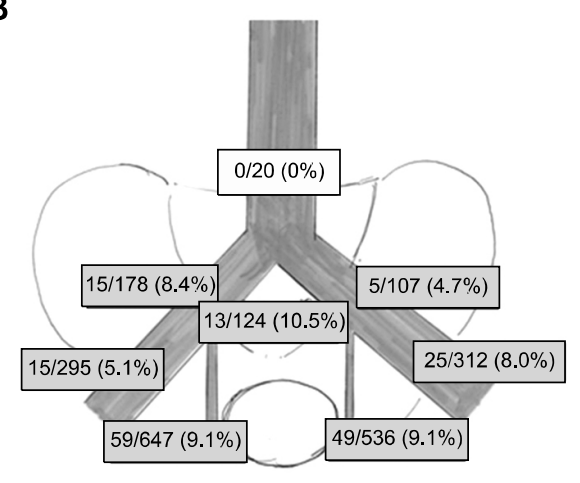

Fig. 1. (A) Ratio of patients with lymph node invasions and (B) ratio of positive to total number of removed lymph nodes according to the region of lymph node dissection in patients treated with radical cystectomy and extended pelvic lymph node dissection.
Table 3. Rate of complications of each subgroup according to method of lymph node dissection by Clavien-Dindo classification

\begin{tabular}{ccc}
\hline Clavien-Dindo classification & SPLND & EPLND \\
\hline I & $18(16.5)$ & $20(39.2)$ \\
II & $61(56.0)$ & $23(45.1)$ \\
III & $25(22.9)$ & $7(13.7)$ \\
IV and V & $5(4.6)$ & $1(2.0)$ \\
p-value & \multicolumn{2}{c}{0.063} \\
\hline
\end{tabular}

Values are presented as number (\%).

SPLND: standard pelvic lymph node dissection, EPLND: extended pelvic lymph node dissection.

(Clavien-Dindo classification grade III or higher) was lower in the ePLND group, but the group had a higher number of low-grade complications (Clavien-Dindo classification grade II or lower). Detailed information on overall complications is summarized in Table 4. The most prevalent complications in the sPLND group were perioperative transfusion, wound dehiscence, and ileus. In the ePLND group, the most prevalent complications were urinary tract infection, wound dehiscence, ileus, and anastomosis site leakage. Furthermore, there were no significant differences in rate of postoperative complication according to the type of urinary diversion in our cohort $(\mathrm{p}=0.486)$ (Table 5).

\section{DISCUSSION}

In the present study, we compared the diagnostic accuracy of ePLND and SPLND in patients with bladder cancer who underwent radical cystectomy with PLND as well as the postoperative complications. The rates of patients who had positive lymph nodes were higher in the ePLND group, but the group showed more favorable clinical and pathologic stages than the
Table 4. Perioperative complications in patients who underwent radical cystectomy with pelvic lymph nodes dissection

\begin{tabular}{lcccc}
\hline \multicolumn{1}{c}{ Variable } & Entire patients & SPLND & EPLND \\
\hline Wound dehiscence & $32(17.9)$ & $20(16.1)$ & $12(21.8)$ \\
Urinary tract infection & $31(17.3)$ & $16(12.9)$ & $15(27.3)$ \\
Ileus & $28(15.6)$ & $20(16.1)$ & $8(14.5)$ \\
Anastomosis site leakage & $16(8.9)$ & $8(6.5)$ & $8(14.5)$ \\
Cardiac problem & $2(1.1)$ & $2(1.6)$ & - \\
Bleeding & $2(1.1)$ & 1 & $(0.8)$ & 1 \\
Transfusion & $48(26.8)$ & $41(33.1)$ & $7(12.7)$ \\
Thromboembolism & $4(2.2)$ & $4(3.2)$ & - \\
Infected lymphocele & $4(2.2)$ & $3(2.4)$ & $1(1.8)$ \\
Fistula & $3(1.7)$ & $2(1.6)$ & $1(1.8)$ \\
Miscellaneous & - & - & - \\
Scrotal edema & $1(0.6)$ & $1(0.8)$ & - \\
Duodenal ulcer perforation & $2(1.1)$ & $1(0.8)$ & $1(1.8)$ \\
Vaginal vault prolapse & $1(0.6)$ & $1(0.8)$ & - \\
Peritonitis & $1(0.6)$ & $1(0.8)$ & - \\
Clostridium difficile colitis & $2(1.1)$ & $2(1.6)$ & - \\
Ureteral stent fracture & $1(0.6)$ & $1(0.8)$ & $1(1.8)$ \\
Rectal injury & $1(0.6)$ & - & \\
\hline
\end{tabular}

Values are presented as number (\%).

SPLND: standard pelvic lymph node dissection, EPLND: extended pelvic lymph node dissection.

Table 5. Rate of complications of each subgroup according to method of urinary diversion by Clavien-Dindo classification

\begin{tabular}{lcc}
\hline $\begin{array}{c}\text { Clavien-Dindo } \\
\text { classification }\end{array}$ & Ileal diversion & $\begin{array}{c}\text { Orthopedic } \\
\text { cystoplasty }\end{array}$ \\
\hline I & $30(25.9)$ & $5(15.6)$ \\
II & $58(50.0)$ & $19(59.4)$ \\
III & $24(20.7)$ & $6(18.8)$ \\
IV and V & $4(3.4)$ & $2(6.3)$ \\
p-value & & 0.486 \\
\hline
\end{tabular}

Values are presented as number (\%). 
sPLND group. We believe that these findings imply the higher nodal staging ability of ePLND compared with sPLND.

The absence of consensus in the optimal extent of PLND is because of the lack of randomized controlled studies on this subject. However, there has been a continuous accumulation of evidence, albeit of low level, showing that ePLND has a more accurate staging ability and superior prognostic effect. ${ }^{5,6}$ Herr et $\mathrm{al}^{5}$ analyzed 268 MIBC patients treated with radical cystectomy and reported a positive association between high number of removed lymph nodes and better prognosis. Konety et al. ${ }^{6}$ also analyzed a large cohort of 1,923 patients from the database of the Surveillance, Epidemiology and End Results program and concluded that the extent of PLND was significantly related with better prognosis in terms of cancer-specific survival (CSS). ${ }^{6}$ The patients in their study who had zero to 3 resected lymph nodes showed significantly worse CSS than those with 3 or more. Holmer et al. ${ }^{16}$ retrospectively analyzed 170 patients who underwent radical cystectomy with extended or nonextended PLND. They observed no significant improvement in CSS, but multivariate analysis showed a significant relationship of superior CSS with prolonged time to recurrence in ePLND. Although the present study did not analyze the patients' postoperative survival, we observed a significantly higher number of collected lymph nodes and higher rate of patients with lymph node invasion in the ePLND group.

Another noteworthy finding from the present study is the pattern of lymph node invasion. Leissner et al. ${ }^{17}$ previously defined three PLND levels: level 1, all lymph nodes below the common iliac bifurcation; level 2, lymphatic system above the common iliac bifurcation and below the aortic bifurcation; level 3 , above the aortic bifurcation. Our studies showed a relatively higher rate of lymph node invasion in level 1 areas (mean, $15.8 \%$ ) than level 2 areas (mean, 5.3\%), and no lymph node invasions were noted in the para-aortic area (level 3). Similar findings were also previously suggested by the study of Abol-Enein et al. ${ }^{18}$ They performed a prospective single-center study with 200 patients who underwent radical cystectomy with ePLND. They found that extrapelvic node invasion was always associated with obturator and/or internal iliac lymph node invasion. They argued that the internal, external, and obturator lymph nodes are the primary lymphatic drainage site from the bladder and are regarded as sentinel lymph nodes in patients with bladder cancer. In our study, among the 34 patients with lymph node invasions, only 3 patients with lymph node in- vasions in the level 2 region did not have nodal metastasis in the level 1 region. We believe that our findings provide valuable information supporting the concept of sentinel nodes of bladder cancer from previous studies.

Only a few studies compared the postoperative complications between ePLND and SPLND in bladder cancer patients treated with radical cystectomy. Brössner et al. ${ }^{19}$ compared the complication rates between ePLND and sPLND in their cohorts of 92 patients. The operation duration was significantly longer in the ePLND group than in the sPLND group $(p<0.01)$. As the low-grade complications were similar in both PLND groups, complications requiring interventions were more prevalent in the ePLND group, but the difference was not significant $(p=0.28)$. They concluded that ePLND did not increase the morbidity of radical cystectomy. Poulsen et al. ${ }^{20}$ also reported that no significant impact from the extent of PLND on mortality or lymphocele formation. Another multicenter study, by Leissner et al., ${ }^{17}$ also pointed that there were no significant adverse side effects related with ePLND. In the present study, we observed that the complication rates were quite comparable between the ePLND and SPLND groups with no significant differences $(\mathrm{p}=0.063)$.

Our study has some limitations. First, although anatomic-pathologic data on lymph node invasion were prospectively collected, medical records were retrospectively reviewed. Therefore, our study may have some inherent bias from its retrospective data analyses. Second, the study included only the Korean population; therefore, care must be taken when applying our results to other racial groups. Most importantly, we could not analyze the survival outcomes based on the extent of PLND. A further study with prospective randomized design is needed to determine the optimal extent of PLND in patients treated with radical cystectomy.

\section{CONCLUSIONS}

The ePLND showed more accurate nodal staging ability than sPLND in patients who underwent radical cystectomy for localized bladder cancer. Moreover, there were no significant differences in complication rates between the 2 groups.

\section{CONFLICT OF INTEREST}

The authors claim no conflicts of interest. 


\section{REFERENCES}

1. Jemal A, Siegel R, Xu J, Ward E. Cancer statistics, 2010. CA Cancer J Clin 2010;60:277-300

2. Stein JP, Lieskovsky G, Cote R, Groshen S, Feng AC, Boyd $\mathrm{S}$, et al. Radical cystectomy in the treatment of invasive bladder cancer: long-term results in 1,054 patients. J Clin Oncol 2001;19:666-75

3. Stenzl A, Cowan NC, De Santis M, Kuczyk MA, Merseburger AS, Ribal MJ, et al. Treatment of muscle-invasive and metastatic bladder cancer: update of the EAU guidelines. Eur Urol 2011;59:1009-18

4. Seiler R, Thalmann GN, Zehnder P. Pelvic lymph node dissection in the context of radical cystectomy: a thorough insight into the connection between patient, surgeon, pathologist and treating institution. Res Rep Urol 2013;5:121-8

5. Herr HW, Faulkner JR, Grossman HB, Natale RB, deVere White R, Sarosdy MF, et al. Surgical factors influence bladder cancer outcomes: a cooperative group report. J Clin Oncol 2004;22:2781-9

6. Konety BR, Joslyn SA, O'Donnell MA. Extent of pelvic lymphadenectomy and its impact on outcome in patients diagnosed with bladder cancer: analysis of data from the Surveillance, Epidemiology and End Results Program data base. J Urol 2003;169:946-50

7. Bi L, Huang H, Fan X, Li K, Xu K, Jiang C, et al. Extended vs non-extended pelvic lymph node dissection and their influence on recurrence-free survival in patients undergoing radical cystectomy for bladder cancer: a systematic review and meta-analysis of comparative studies. BJU Int 2014;113(5b): E39-48

8. Poulsen AL, Horn T, Steven K. Radical cystectomy: extending the limits of pelvic lymph node dissection improves survival for patients with bladder cancer confined to the bladder wall. J Urol 1998;160(6 Pt 1):2015-9

9. Dhar NB, Klein EA, Reuther AM, Thalmann GN, Madersbacher S, Studer UE. Outcome after radical cystectomy with limited or extended pelvic lymph node dissection. J Urol 2008;179:873-8

10. Jensen JB, Ulhøi BP, Jensen KM. Extended versus limited lymph node dissection in radical cystectomy: impact on recurrence pattern and survival. Int J Urol 2012;19:39-47

11. Simone G, Papalia R, Ferriero M, Guaglianone S, Castelli E, Collura D, et al. Stage-specific impact of extended versus standard pelvic lymph node dissection in radical cystectomy. Int J Urol 2013;20:390-7

12. Tilki D, Brausi M, Colombo R, Evans CP, Fradet Y, Fritsche $\mathrm{HM}$, et al. Lymphadenectomy for bladder cancer at the time of radical cystectomy. Eur Urol 2013;64:266-76

13. Greene FL, Page DL, Fleming ID, Fritz AG, Balch CM, Haller DG, et al., editors. AJCC cancer staging manual. 6th ed. New York: Springer-Verlag; 2002

14. Epstein JI, Amin MB, Reuter VR, Mostofi FK. The World Health Organization/International Society of Urological Pathology consensus classification of urothelial (transitional cell) neoplasms of the urinary bladder. Bladder Consensus Conference Committee. Am J Surg Pathol 1998;22:1435-48

15. Clavien PA, Barkun J, de Oliveira ML, Vauthey JN, Dindo $\mathrm{D}$, Schulick RD, et al. The Clavien-Dindo classification of surgical complications: five-year experience. Ann Surg 2009; 250:187-96

16. Holmer M, Bendahl PO, Davidsson T, Gudjonsson S, Månsson W, Liedberg F. Extended lymph node dissection in patients with urothelial cell carcinoma of the bladder: can it make a difference? World J Urol 2009;27:521-6

17. Leissner J, Ghoneim MA, Abol-Enein H, Thüroff JW, Franzaring L, Fisch M, et al. Extended radical lymphadenectomy in patients with urothelial bladder cancer: results of a prospective multicenter study. J Urol 2004;171:139-44

18. Abol-Enein H, El-Baz M, Abd El-Hameed MA, Abdel-Latif M, Ghoneim MA. Lymph node involvement in patients with bladder cancer treated with radical cystectomy: a patho-anatomical study--a single center experience. J Urol 2004;172(5 Pt 1):1818-21

19. Brössner C, Pycha A, Toth A, Mian C, Kuber W. Does extended lymphadenectomy increase the morbidity of radical cystectomy? BJU Int 2004;93:64-6

20. Poulsen AL, Horn T, Steven K. Radical cystectomy: extending the limits of pelvic lymph node dissection improves survival for patients with bladder cancer confined to the bladder wall. J Urol 1998;160(6 Pt 1):2015-9 